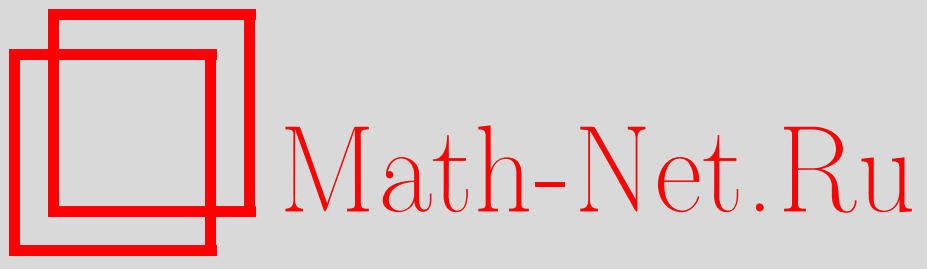

С. А. Вугальтер, Г. М. Жислин, О дискретном спектре гамильтонианов атомов в однородном магнитном поле, Функи. анализ и его прил., 2000, том 34, выпуск 1, 80-83

DOI: https://doi.org/10.4213/faa285

Использование Общероссийского математического портала MathNet.Ru подразумевает, что вы прочитали и согласны с пользовательским соглашением http://www.mathnet.ru/rus/agreement

Параметры загрузки:

IP : 54.210 .77 .194

26 апреля 2023 г., 14:34:25

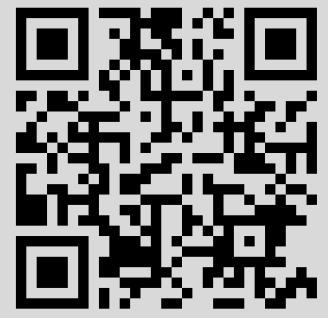




\title{
О дискретном спектре гамильтонианов атомов в однородном магнитном поле*
}

\author{
(c) 2000. С. А. ВугАльтеР, Г. М. ЖКИСлин
}

1. Рассмотрим оператор Шрёдингера $n$-электронного атома в однородном магнитном поле

$$
H(B)=m_{0}^{-1}\left(i \nabla_{0}+n A_{0}\right)^{2}+\sum_{j=1}^{n}\left(i \nabla_{j}-A_{j}\right)^{2}-n \sum_{j=1}^{n}\left|r_{j}-r_{0}\right|^{-1}+\sum_{\substack{s, t=1 \\ s<t}}^{n}\left|r_{s}-r_{t}\right|^{-1},
$$

где $m_{0}$ и $r_{0}=\left(x_{0}, y_{0}, z_{0}\right)$ - масса и координаты ядра, $r_{i}=\left(x_{i}, y_{i}, z_{i}\right), i=$ $1, \ldots, n,-$ координаты электронов, $A_{j}=\left(-y_{j}, x_{j}, 0\right) B, \nabla_{j}=\left(\partial / \partial x_{j}, \partial / \partial y_{j}\right.$, $\left.\partial / \partial z_{j}\right), j=0,1, \ldots, n$, и $B>0$ - магнитная индукция; заряд и масса электрона приняты соответственно за -1 и +1 .

При $B=0$ (атом в отсутствии магнитного поля) оператор допускает отделение движения центра масс, и полученный после такого отделения оператор $H(0)$ имеет непустой дискретный спектр [1]. С физической точки зрения это является доказательством устойчивости атомов как многоэлектронных систем.

При $B \neq 0$ движение центра масс может быть отделено только в направлении поля. Обозначим полученный после такого отделения оператор через $H_{o z}(B)$. Для него неверна теорема Хунцикера-Ван Винтера-Жислина (ХВЖ) [6] о локализации существенного спектра, что затрудняет спектральный анализ.

2. Для изучения спектра оператора $H_{o z}(B)$ в [2] предложен метод фиксации псевдомомента, заменяющий в известной степени отделение движения центра масс в плоскости $(x, y)$. Оператор псевдомомента имеет вид

$$
P=\left(P_{x}, P_{y}\right), \quad P_{x}=\sum_{j=0}^{n}\left(\frac{1}{i} \frac{\partial}{\partial x_{j}}-e_{j} B y_{j}\right), \quad P_{y}=\sum_{j=0}^{n}\left(\frac{1}{i} \frac{\partial}{\partial y_{j}}+e_{j} B x_{j}\right),
$$

где $e_{0}=n, e_{j}=-1, j \geqslant 1$.

Оператор $H_{o z}(B)$ коммутирует с $P$, и, поскольку рассматриваемая система нейтральна, $P_{x} P_{y}=P_{y} P_{x}[2]$. Следовательно, оператор $H_{o z}(B)$ можно редуцировать на подпространство функций с фиксированными значениями компонент псевдомомента $P_{x}=\nu_{1}, P_{y}=\nu_{2}$.

Обозначим полученный при этом оператор, действующий в пространстве $\mathbb{R}^{3 n}$, через $H_{\nu}(B)$ (выражение для $H_{\nu}(B)$ при произвольном $n$ см. в [3]; мы не приводим его здесь из-за громоздкости. В частном случае $n=3$ явный вид оператора $H_{\nu}(B)$ дается далее равенством $\left.(2)\right)$.

Операторы $H_{o z}(B)$ и $H_{\nu}(B)$ связаны соотношением

$$
H_{o z}(B)=\int H_{\nu}(B) d \nu,
$$

и, как было показано в [2] (см. также [3]), для гамильтониана $H_{\nu}(B)$ верна ХВЖ-теорема. Согласно ей, нижняя грань $\mu_{\nu}$ существенного спектра

\footnotetext{
*Работа поддержана грантами РФФИ 96-01-00478 и ИНТАС 95-0414.
} 
$\sigma_{\text {ess }}\left(H_{\nu}(B)\right)$ оператора $H_{\nu}(B)$ совпадает с нижней гранью оператора $\widehat{H}_{\nu}(B)$, определяемого следующим образом. Рассмотрим оператор $\widehat{H}_{o z}(B)$ (действующий в пространстве $\mathbb{R}^{3 n+1}$ ), который получается из оператора (1) удалением из него всех членов, содержащих координату $z_{n}$, и отделением в полученном таким образом операторе движения центра масс системы $\{0,1, \ldots, n-1\}$ в направлении поля. Оператор $\widehat{H}_{\nu}(B)$ есть редуцированный на подпространство состояний с фиксированным псевдомоментом оператор $\widehat{H}_{o z}(B)$. Оператор $\widehat{H}_{\nu}(B)$ действует в пространстве $\mathbb{R}^{3 n-1}$. Его явный вид в обшем случае см. в [3]; при $n=3$ выражение для $\widehat{H}_{\nu}(B)$ дано ниже равенством $(3)$.

3. В недавних работах $[4,5]$ была получена асимптотика дискретного спектра $(n+1)$-частичного оператора $H_{\nu}(B)$ в предположении, что число $\mu_{\nu}$ есть точка дискретного спектра $\sigma_{d}\left(\widehat{H}_{\nu}(B)\right)$ оператора $\widehat{H}_{\nu}(B)$. Однако это предположение было доказано только для одноэлектронных и двухэлектронных систем (атомы водорода и гелия). Поэтому вопрос о структуре спектра $\sigma_{d}\left(H_{\nu}(B)\right)$ при $n \geqslant 3$ оставался открытым.

В настоящей работе включение $\mu_{\nu} \in \sigma_{d}\left(\widehat{H}_{\nu}(B)\right)$ доказывается для любого $n$. Таким образом, с учетом результатов $[4,5]$ мы получаем теорему о структуре спектра $\sigma_{d}\left(H_{\nu}(B)\right)$ для любых атомов, не содержащую никаких условий и предположений.

Пусть $N\left(\mu_{0}-\lambda\right)$ - размерность линейной оболочки собственных функций оператора $\widehat{H}_{\nu}(B)$, отвечающих его собственным значениям, не превосходящим $\mu_{0}-\lambda$.

ТЕОремА. Для всех значений псевдомомента $\nu=\left(\nu_{1}, \nu_{2}\right)$ дискретный спектр оператора $H_{\nu}(B)$ бесконечен и

$$
\lim _{\lambda \rightarrow 0+0} N\left(\mu_{\nu}-\lambda\right) \lambda^{1 / 2}=d n\left(\frac{m_{0}+n-1}{m_{0}+n}\right)^{1 / 2} \frac{\pi}{2},
$$

где $d$ - размерность собственного подпространства оператора $\widehat{H}_{\nu}(B)$, отвечающего числу $\mu_{\nu}$.

ЗАмечАнИЕ. Отметим, что при $B=0$ выполняется соотношение $N(\mu-\lambda) \sim$ $\lambda^{-3 / 2}$. При $B \neq 0$ и фиксации псевдомомента система в магнитном поле подобна одномерной (а не трехмерной, как в отсутствии поля) частице в эффективном поле, убывающем, как $|\eta|^{-1}, \eta \in \mathbb{R}^{1}$.

4. Опишем основные идеи доказательства для случая $n=3$ (атом лития), так как при $n>3$ дополнительных трудностей не возникает. Отметим, что все частицы, кроме ядра, являются электронами, а для тождественных частиц возможно отделение движения центра масс в плоскости, перпендикулярной направлению поля. Кроме того, в силу равенства зарядов электронов оператор псевдомомента не зависит от относительных координат электронов, а зависит только от положений ядра и центра масс электронной подсистемы. Эти два обстоятельства приводят к тому, что кинетическая часть оператора $H_{\nu}(B)$, отвечающая движению в плоскости, перпендикулярной полю, может быть представлена как сумма соответствующего оператора для эффективной двухчастичной подсистемы (состоящей из ядра и эффективной частицы) с фиксированным псевдомоментом и оператора относительного движения электронов. Такое 
представление позволяет показать, что граница существенного спектра этого оператора есть дискретное собственное значение соответствующего оператора $\widehat{H}_{\nu}(B)$, и тем самым доказать теорему.

Пусть $x_{c}=\frac{1}{3} \sum_{j=1}^{3} x_{j}, y_{c}=\frac{1}{3} \sum_{j=1}^{3} y_{j}, t_{1}=x_{0}-x_{c}+\nu_{1} /(2 B), t_{2}=y_{0}-y_{c}-$ $\nu_{2} /(2 B), r_{j \perp}=\left(x_{j}, y_{j}\right), m_{R}^{-1}=\left(m_{0}-3\right) /\left(3 m_{0}\right), q_{\perp}=\left(q_{1 \perp}, q_{2 \perp}\right)=r_{2 \perp}-r_{3 \perp}, \zeta_{\perp}=$ $\left(\zeta_{1 \perp}, \zeta_{2 \perp}\right)=\frac{1}{2}\left(r_{2 \perp}+r_{3 \perp}\right)-r_{1 \perp}{ }^{1}$. В новых переменных аналогично соотношению (3.2) из [3] имеем

где

$$
H_{\nu}(B)=-\Delta_{o z}+T_{\perp}(\nu ; B)-\sum_{j=1}^{3} 3\left|r_{0}-r_{j}\right|^{-1}+\sum_{i<j}^{1,3}\left|r_{i}-r_{j}\right|^{-1},
$$

$$
\begin{aligned}
T_{\perp}(\nu ; B)=\left(i \nabla_{q_{\perp}}+A_{q_{\perp}}\right)^{2}+\left(i \nabla_{\zeta_{\perp}}+A_{\zeta_{\perp}}\right)^{2}-2 m_{0}^{-1}\left(\frac{\partial^{2}}{\partial t_{1}^{2}}+\frac{\partial^{2}}{\partial t_{1}^{2}}\right) \\
\quad+m_{R}^{-1}\left\{\left(i \frac{\partial}{\partial t_{1}}+3 B t_{2}\right)^{2}+\left(i \frac{\partial}{\partial t_{2}}-3 B t_{1}\right)^{2}\right\}+2 m_{0}^{-1}\left(t_{1}^{2}+t_{2}^{2}\right), \\
\nabla_{\eta \perp}=\left(\frac{\partial}{\partial \eta_{1}}, \frac{\partial}{\partial \eta_{2}}\right), \quad A_{\eta \perp}=B\left(-\eta_{2}, \eta_{1}\right), \quad \eta=\left(\eta_{1}, \eta_{2}\right)=q_{\perp}, \zeta_{\perp}
\end{aligned}
$$

a $-\Delta_{o z}$ - оператор кинетической энергии относительного движения системы вдоль направления поля.

Мы уже говорили, что, согласно $[2,3], \mu_{\nu}=\inf \sigma_{\mathrm{ess}}\left(H_{\nu}(B)\right)=\inf \widehat{H}_{\nu}(B)$, где оператор $\widehat{H}_{\nu}(B)$ в общем случае определен в п. 2. Для атома лития (т.е. для оператора $(2))$ мы можем записать оператор $\widehat{H}_{\nu}(B)$ в виде

$$
\widehat{H}_{\nu}(B)=-\widehat{\Delta}_{o z}+T_{\perp}(\nu ; B)-\sum_{j=1}^{2} 3\left|r_{0}-r_{j}\right|^{-1}+\left|r_{1}-r_{2}\right|^{-1},
$$

где $-\widehat{\Delta}_{o z}$ - оператор кинетической энергии относительного движения вдоль оси $z$ для подсистемы $(0,1,2)$.

5. Легко видеть, что энергия системы неограниченно возрастает с ростом $\left|t_{1}\right|+\left|t_{2}\right|$. Поэтому и в силу фиксации величин $\nu_{1}, \nu_{2}$ компонент псевдомомента состояния системы с большим расстоянием между ядром и центром масс электронной подсистемы в плоскости, перпендикулярной полю, не могут принадлежать ее дискретному спектру. Отсюда следует, что состояния рассматриваемой системы, порождаемые уходом одного электрона от остальных и ядра в плоскости $(x, y)$, также не могут принадлежать ее дискретному спектру. Основная трудность, преодоленная в данной работе, состоит в доказательстве того факта, что нижний предел значений энергии по последовательности волновых функций, отвечающей уходу из системы двух электронов, принадлежит существенному спектру. Отсюда следует, что дискретный спектр (исключая, быть может, конечное число точек) может отвечать только уходу одного электрона в направлении оси $z$. Поэтому асимптотика дискретного спектра имеет одномерный характер.

${ }^{1}$ Мы не учитываем ради простоты перестановочную симметрию системы. Чтобы учесть ее, новые координаты электронов надо вводить инвариантным образом - относительно центра масс электронов: $q_{\perp}=r_{2 \perp}-r_{c \perp}, \zeta_{\perp}=r_{3 \perp}-r_{c \perp}$, где $r_{c \perp}=\left(x_{c}, y_{c}\right)$. 


\section{ЛИТЕРАТУРА}

1. Жислин Г. M. Труды MMO, 9, 81-120 (1960). 2. Avron J. E., Herbst J. W., Simon B. Ann. Phys., 114, 431 (1978). 3. Вугальтер С. А., Жислин Г. М. ТМФ, 113, No. 3, 413-431 (1997). 4. Zhislin G. The spectrum of the relative motion of many-particle systems in magnetic fields: what do we know about it? In: A tribute to Michail S. Birman, Amer. Math. Soc., 1998, pp. 275-285. 5. Жислин Г. М. Спектральные свойства гамильтонианов с магнитным полем при фиксации псевдомомента. II. ТМФ, 118, № 1 , 15-39 (1999). 6. Рид М., Саймон Б. Методы современной математической физики. 4. Анализ операторов. Мир, М., 1982.

Нижегородский научно-исследовательский

Поступило в редакцию радиофизический институт

17 апреля 1998 г.

\section{УДК 517.9}

\section{Бифуркации критических торов для функционалов с 3-круговой симметрией}

(C) 2000. А. В. ГНЕЗдилов

В изучении фазовых переходов в сегнетоэлектриках (см. $[1,2])$ важную роль играют бифуркации множеств критических точек гладкого функционала, заданного на банаховом многообразии $M$ и инвариантного относительно гладкого действия $m$-мерного тора. Функционалу, допускающему конечномерную эквивариантную редукцию на некоторой области из $M$, сопоставляется гладкая функция на конечномерном пространстве (называемом пространством ключевых переменных), инвариантная относительно некоторого действия тора на этом пространстве (см. [3]).

Рассмотрим стандартное действие $m$-мерного тора на $\mathbb{C}^{m}$ покоординатным умножением на комплексные числа, равные по модулю единице. Функция $W$ на $\mathbb{R}^{2 m} \approx \mathbb{C}^{m}$, инвариантная относительно этого действия, называется функцией с $m$-круговой симметрией. Такой функции отвечает гладкая функция $\widetilde{W}$ на факторпространстве, изоморфном положительному координатному углу в $\mathbb{R}^{m}$.

Угловые особенности функций изучались Д. Сирсмой [4] как обобщения краевых особенностей В. И. Арнольда [5]. Простейшая невырожденная морсовская особая точка в нуле функции на $\mathbb{R}^{m}$, рассматриваемая как угловая особенность, называется полурегулярной.

Бифуркационные диаграммы $\Sigma$ полурегулярных угловых особенностей изучались в [6]. Однако нужный для приложений вопрос о перечислении компонент дополнения к такой бифуркационной диаграмме, т.е. вопрос о перечислении всех возможных наборов (называемых ниже bif-раскладами) критических точек сужений функции $\widetilde{W}$ на грани угла, или, другими словами, наборов невырожденных критических орбит разных размерностей функции $W$ с поликруговой симметрией, возникающих при возмущениях общего положения полурегулярной особенности, при $m \geqslant 3$ оставался открытым до сих пор. Настоящая заметка решает этот вопрос при $m=3$ и, в частности, дает полное описание бифуркации морсовских критических орбит из точки минимума с особенностью типа 3-круговой сборки. 